\title{
Ultrasound in the diagnosis of diaphragmatic paralysis after operation for congenital heart disease
}

Seshadri Balaji, Pavel Kunovsky, Ian Sullivan

\begin{abstract}
Phrenic nerve palsy is a recognised complication of operation for congenital heart disease in children. The accuracy of ultrasound in assessing diaphragmatic motion was prospectively compared with fluoroscopy in 16 patients in whom phrenic nerve palsy was suspected. Ultrasound successfully identified the five patients with phrenic nerve palsy; there were no false positive or false negative diagnoses.

Ultrasound was as effective as fluoroscopy in the diagnosis of abnormalities of diaphragmatic motion.
\end{abstract}

Diaphragmatic paralysis due to phrenic nerve injury may complicate as many as $10 \%$ of operations for complex congenital heart disease. ${ }^{1}$ Though spontaneous recovery can be expected in about $85 \%$ of patients, it is associated with considerable morbidity, especially in children under the age of two years. ${ }^{1}$ Early diagnosis and, when appropriate, surgical plication of the affected hemidiaphragm helps to reduce the time spent in intensive care and the duration of ventilatory assistance and is therefore an important part of the management. ${ }^{2}$

Fluoroscopy is the standard method of assessing diaphragmatic motion. Fluoroscopy, however, has disadvantages. The patient and staff are exposed to radiation. Also the patient, often still on ventilatory support, has to be moved to the fluoroscopy screening room with monitoring and infusion lines in place. This is potentially dangerous as well as being time and labour consuming. In contrast, ultrasound scanning with portable equipment can identify abnormalities of diaphragmatic motion at the bedside. We therefore prospectively studied patients with suspected diaphragmatic palsy after operation for congenital heart disease by both techniques to compare their diagnostic accuracy.

Department of
Cardiology, The
Hospital for Sick
Children, London
S Balaji
P Kunovsky
I Sullivan
Correspondence to
Dr Seshadri Balaji,
Department of Cardiology,
The Hospital for Sick
Children, Great Ormond
Street, London WCIN 3JH.
Accepted for publication
22 March 1990

\section{Patients and methods}

PATIENTS

Between 1 August and 1 December 1989, diaphragmatic palsy was suspected in 16 patients, aged 1 day to 8 years (median 8 months) from a total of 228 children who underwent operation for congenital heart disease at the Hospital for Sick Children, London. Indicators of possible diaphragmatic
Table 1 Operations in children with suspected diaphragmatic palsy

\begin{tabular}{ll}
\hline Operation & Number \\
\hline Repair of tetralogy of Fallot & 5 \\
Pulmonary artery banding & 2 \\
AVSD repair & 2 \\
Right MBTS & 1 \\
Truncus arteriosus repair & 1 \\
Fontan procedure & 1 \\
PA,IVS: PV, left MBTS & 1 \\
DORV repair & 1 \\
RPA from aorta repair & 1 \\
Resection of coarctation & 1
\end{tabular}

AVSD, complete atrioventricular septal defect; DORV, double outlet right ventricle; PA,IVS, pulmonary atresia, intact ventricular septum; PV, pulmonary valvotomy; RPA, right pulmonary artery; MBTS, modified Blalock-Taussig shunt.

palsy were failure to be weaned from assisted ventilation at a time considered appropriate for the child, a raised hemidiaphragm on the chest radiograph, an asymmetric breathing pattern, or recurrent unilateral lung collapse. Table 1 lists the operations performed on these patients. The time between operation and diaphragmatic screening ranged from 2 to 24 days (mean (SD) 8 (6) days).

\section{METHODS}

Ultrasound assessment was performed by one investigator (SB) without knowledge of the results of fluoroscopic examination, which was performed by a different operator on the same day. An Advanced Technology Laboratories (ATL) Ultramark 4 ultrasound scanning machine was used with $7 \cdot 5,5$, or $3 \mathrm{MHz}$ mechanical sector scanheads as appropriate to the size of the child. Subcostal views (fig 1) with the transducer angled superiorly and posteriorly from the subxiphoid region at the midline, parasagittal views with the transducer at the lower costal margin in the midclavicular line, and coronal views (fig 2) with the transducer at the mid-axillary line were obtained in each case. Fluoroscopy was performed by anteroposterior screening of the thorax in the cardiac catheterisation laboratory.

All patients except one were on ventilatory assistance at the time of assessment. The ventilator was turned to the continuous positive airways pressure mode during the brief time required for each examination. The phases of respiration were timed by the operator placing a hand on the patient's chest during the examination. Paralysis of a hemidiaphragm was diagnosed if there was upward movement or absence of movement during inspiration (fig 3). 

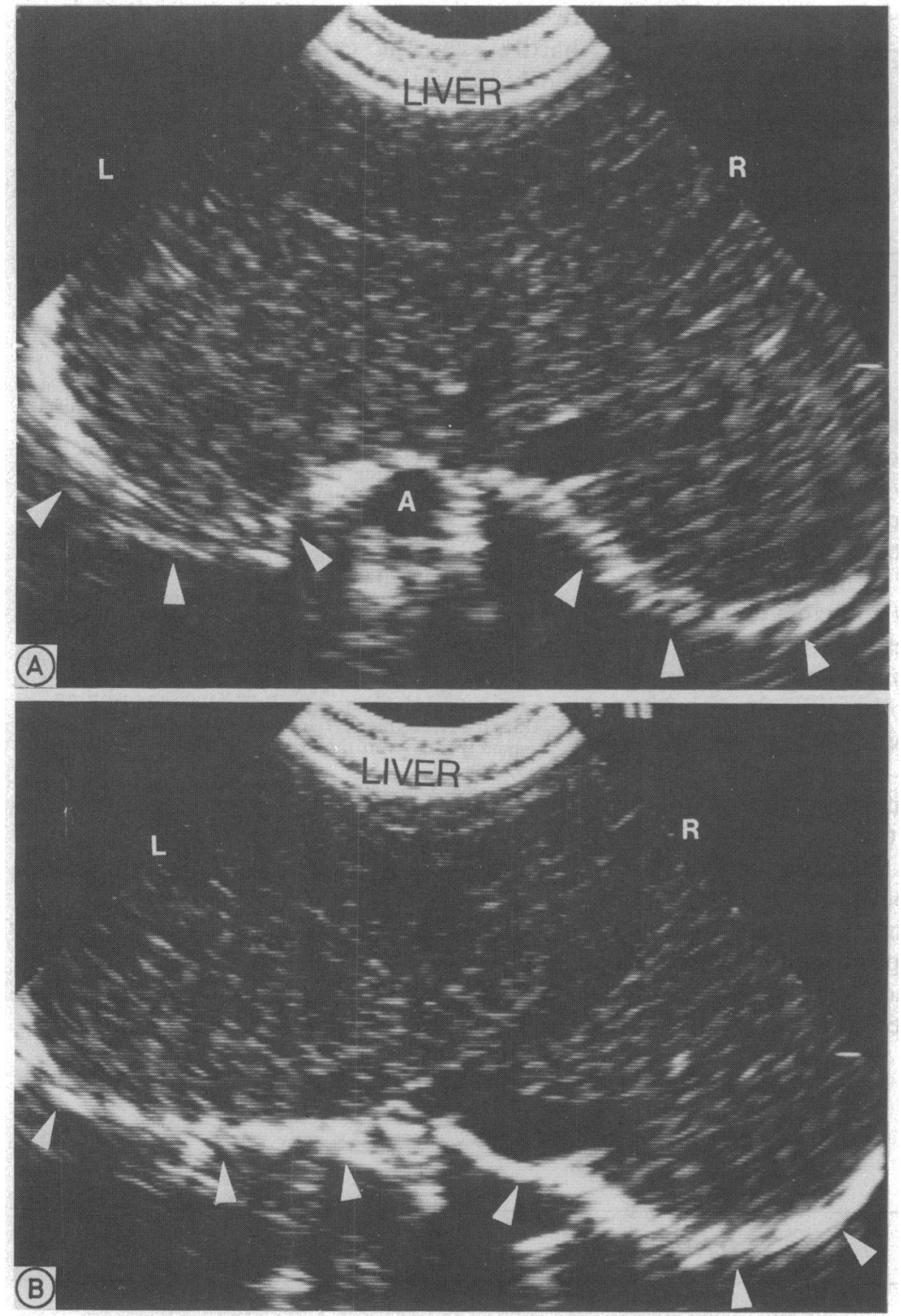

Figure 1 Subcostal view of the two hemidiaphragms (arrowheads) in expiration ( $A$ ) and inspiration $(B)$. Note that both hemidiaphragms have moved downwards (closer to the transducer) and appear flatter. A, aorta.

\section{Results}

Ultrasound imaging from the subcostal position (fig 1) provided satisfactory imaging of both hemidiaphragms in all but the largest patients. Using a combination of parasagittal and coronal (fig 2 ) views we were able to assess diaphragmatic motion in these patients.

Paralysis of the left hemidiaphragm was

Figure 2 Coronal view of the right hemidiaphragm (arrowheads). Liver is to the left and right lung (RL) to the right. Arrow at top left points towards the head.

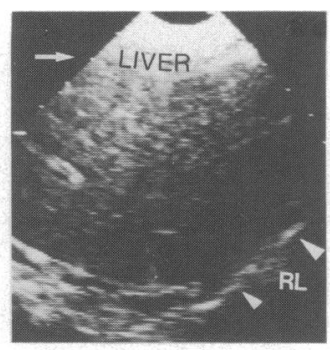

Discussion

Ultrasound assessment of diaphragmatic

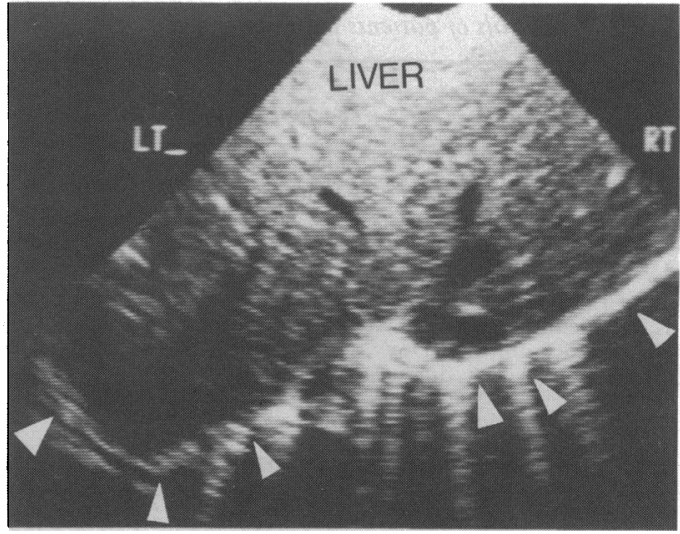

Figure 3 Subcostal view from a patient (case 1, table 2) with paralysed left hemidiaphragm. The right hemidiaphragm has contracted and is flat while the left side has moved upwards-there is paradoxical motion away from the transducer.

motion has been reported previously. Haber and coworkers studied 110 adults with intraabdominal abnormalities and commented on the usefulness of ultrasound in visualising diaphragmatic motion. ${ }^{3}$ There have also been case reports on the use of ultrasound in children with juxtadiaphragmatic lesions, ${ }^{4}$ eventration, ${ }^{5}$ and paralysis of the diaphragm. ${ }^{6}$ Harris and colleagues quantitatively studied the ventilatory movement of the right hemidiaphragm in 50 healthy adults ${ }^{7}$ and a similar study was performed in infants. ${ }^{8}$ Diament et al used ultrasound in a heterogeneous group of 14 children with suspected abnormalities of diaphragmatic motion and diagnosed paralysis of a hemidiaphragm in eight of them. ${ }^{9}$ Watanabe and colleagues, in a large retrospective study of 125 patients with paralysed hemidiaphragm, reported that ultrasound was the only technique used to establish the diagnosis in 14 cases and ultrasound combined with fluoroscopy was used in a further 10 cases. ${ }^{1}$ Nevertheless, fluoroscopy has remained the investigation of choice in the assessment of suspected diaphragmatic palsy in most units.

Diagnosis of diaphragmatic palsy may be delayed by reluctance to undertake a procedure that requires a child on ventilatory and inotropic support to be moved from the intensive care unit to the fluoroscopy room. Furthermore, fluoroscopy delivers an appreciable dose of radiation. In contrast, ultrasound equipment is available in all units that regularly perform cardiac surgery and it can be easily brought to the patient's bedside. Ultrasound is not known to have any dangerous side effects. Prompt diagnosis of diaphragmatic palsy is important because in some patients early plication of the affected hemidiaphragm can significantly reduce morbidity. ${ }^{2}$

Both techniques rely on the patient's capacity to breathe and therefore they cannot be applied to patients on full ventilatory support. Studies of phrenic nerve conduction ${ }^{10}$ are likely to be the only method of diagnosis in this group but not many people are skilled in this technique. Also, to be of any advantage, such studies would have to be used routinely after 
Table 2 Details of patients with confirmed diaphragmatic palsy

\begin{tabular}{llllll}
\hline $\begin{array}{l}\text { Patient } \\
\text { No }\end{array}$ & Age & Diagnosis & Operation & Result & Outcome \\
\hline 1 & 15 months & Fallot & Repair & L Palsy & - \\
2 & 3 years & Fallot, LMBT, WS & Repair & L Palsy & Plication \\
3 & 8 months & DS, AVSD & PAB & L Palsy & Plication \\
4 & 16 months & Fallot & Repair & L Palsy & Plication \\
5 & 22 months & Fallot, DA & Repair & L Palsy & - \\
\hline
\end{tabular}

L, left hemidiaphragm; LMBT, previous left modified Blalock-Taussig shunt; WS, previous Waterston shunt; DS, Down syndrome; AVSD, atrioventricular septal defect; PAB, pulmonary artery banding; DA, ductus arteriosus.

usually suspected until there is difficulty weaning a patient off artificial ventilation.

We found that ultrasound examination at the bedside was as effective as fluoroscopy in the diagnosis of a paralysed hemidiaphragm. Bedside examination facilitates early diagnosis and is of particular benefit in infants, when plication is often necessary to achieve extubation. There were no cases of paralysed right hemidiaphragm in our group, but we would not expect the diagnosis to be difficult with ultrasound because it is generally easier to image the right hemidiaphragm than the left. Bilateral palsy can be missed on fluoroscopic examination unless careful attention is given to the phases of respiration, because the two sides move in concert-albeit paradoxically. This error is less likely with ultrasound assessment because each hemidiaphragm is imaged independently. Ultrasound imaging of the left hemidiaphragm can be difficult in bigger chil- dren and adults, but diaphragmatic palsy is hardly ever the cause of major respiratory morbidity in this group and they rarely require plication.

We thank Dr Ian James, Dr Edward Sumner, and Dr Duncan Macrae of the Cardiac Intensive Care Unit for allowing us to study patients under their care.

Watanabe T, Trusler GA, Williams WG, Edmonds JF, Coles JG, Hosokawa Y. Phrenic nerve paralysis after paediatric cardiac surgery. Retrospective study of 125 cases. J Thorac Cardiovasc Surg 1987;94:383-8.

2 Affatato A, Villagra F, De Leon JP, et al. Phrenic nerve paralysis following paediatric cardiac surgery. Role of diaphragmatic plication. J Cardiovasc Surg 1988;29: 606-9.

3 Haber K, Asher WM, Freimanis AK. Echographic evaluation of diaphragmatic motion in intra-abdominal diseases. Radiology 1975;114:141-4.

4 Kangarloo H, Sukov R, Sample WF, Lipson M, Smith L. Ultrasonographic evaluation of juxtadiaphragmatic masses in children. Radiology 1977;125:785-7.

5 Haller JO, Waldroup LD. Sonographic evaluation of the chest in infants and children. Am J Radiol 1980;134:1019-27.

6 Aldrich TK, Herman JH, Rochester DF. Bilateral diaphrag matic paralysis in the new born infant. J Pediatr 1980; matic paralys

7 Harris RS, Giovannetti M, Kim BK. Normal ventilatory movement of the right hemidiaphragm studied by ultrasonography and pneumotachography. Radiology 1983; 146:141-4.

8 Laing IA, Teele RL, Stark AR. Diaphragmatic movement in newborn infants. J Pediatr 1988;112:638-43.

9 Diament MJ, Boechat MI, Kangarloo H. Real-time sector ultrasound in the evaluation of suspected abnormalities of diaphragmatic motion. J Clin Ultrasound 1985;13:539-43.

10 Mok Q, Ross-Russell R, Shinebourne EA. Prospective study of the frequency of phrenic nerve paralysis after cardiac surgery in children [Abstract]. Br Heart J 1989;61:99.

\section{VIEWS FROM THE PAST Ernest Henry Starling}

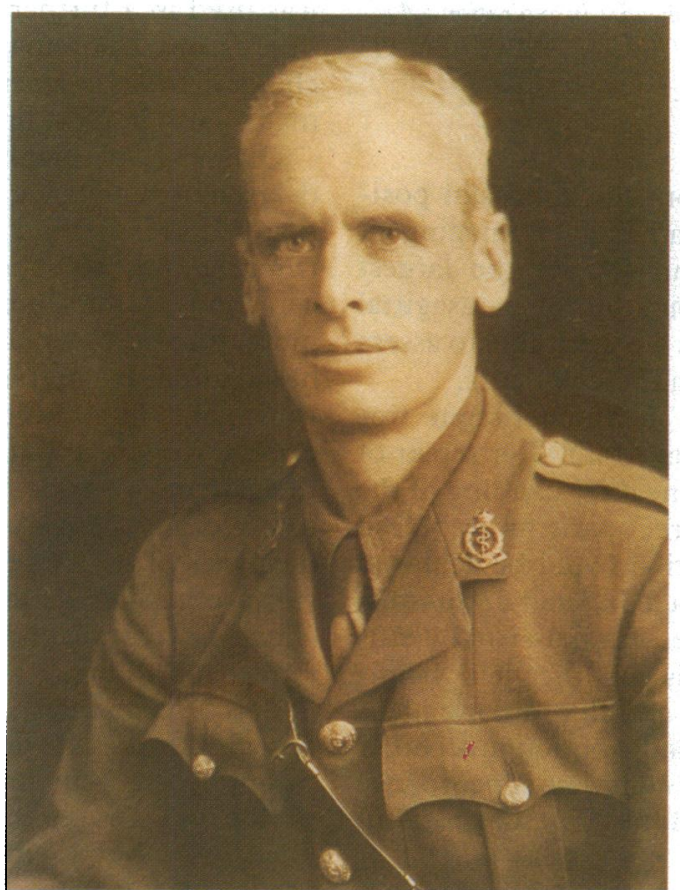

Starling had already published his law of the heart (see page 4) before his military service during the first world war. Using cardiac catheterisation, John McMichael, Peter Sharpey-Schafer, and Sheila Howarth questioned the general applicability of this law in studies started during the second world war (Lewis collection). 